June 1991

QMW/PH/ 91/13

\title{
Hamiltonian Construction of W-gravity Actions
}

\author{
Aleksandar Miković円] \\ Department of Physics, Queen Mary and Westfield College, \\ Mile End Road, London E1 4NS, U.K.
}

\begin{abstract}
We show that all W-gravity actions can be easilly constructed and understood from the point of view of the Hamiltonian formalism for the constrained systems. This formalism also gives a method of constructing gauge invariant actions for arbitrary conformally extended algebras.
\end{abstract}

\footnotetext{
${ }^{1}$ Work supported by the U.K. Science and Engineering Research Council

${ }^{2}$ E-mail address: MIKOVIC@V1.PH.QMW.AC.UK
} 
Actions for a large class of W-gravity theories have been constructed so far [1-6]. The method which was used for the construction of the gauge invariant actions in all these cases was the Noether method and it's generalization in terms of the $F_{ \pm}$ auxiliary fields. It was pointed out in [6] that the $F$ fields satisfy a formal bracket algebra reminiscent of a Poisson bracket algebra in a Hamiltonian formalism where both $x^{+}=\frac{1}{\sqrt{2}}\left(x^{0}+x^{1}\right)$ and $x^{-}=\frac{1}{\sqrt{2}}\left(x^{0}-x^{1}\right)$ are regarded as evolution parameters. In the chiral case one can take $x^{-}$(or $x^{+}$, which depends on the chirality of the currents) to be the evolution parameter, and the usual Hamiltonian interpretation emerges [6]. A natural question then arises in the non-chiral case. What is the first order Hamiltonian form of a W-gravity action? In this letter we give the answer to this question, and furthermore, due to the simplicity of the answer, we propose an alternative way to that of reference [6] of constructing gauge invariant actions for arbitrary conformally extended algebras.

First we review those aspects of the Hamiltonian formalism which will be needed for our construction. Let $\left(p_{i}(t), q^{i}(t)\right)$ be primary canonical variables of a dynamical system with the Hamiltonian $H_{0}(p, q)$. Let $G_{\alpha}(p, q)$ and $\Theta_{\mu}(p, q)$ be the first and the second class constraints, respectively. $t$ is the time, and the indices $i, \alpha, \mu$ can take both the discrete and the continious values, and can be bosonic or fermionic. The Poisson bracket is defined as

$$
\{A, B\}=\frac{\partial_{L} A}{\partial p_{i}} \frac{\partial_{R} B}{\partial q^{i}}-(-1)^{\epsilon(A) \epsilon(B)} \frac{\partial_{L} B}{\partial p_{i}} \frac{\partial_{R} A}{\partial q^{i}}
$$

where $\partial_{L}$ and $\partial_{R}$ are left and right derivatives, while $\epsilon(X)=0,1$ if $X$ is a boson, fermion, respectively. Presence of the second class constraints requires the Dirac bracket

$$
\{A, B\}_{D}=\{A, B\}-\left\{A, \Theta_{\mu}\right\}\left(\Delta^{-1}\right)^{\mu \nu}\left\{\Theta_{\nu}, B\right\}
$$

where $\Delta_{\mu \nu}=\left\{\Theta_{\mu}, \Theta_{\nu}\right\}$. The first class constraints generate the gauge symmetries of the dynamical system and satisfy

$$
\begin{gathered}
\left\{G_{\alpha}, G_{\beta}\right\}_{D}=f_{\alpha \beta}{ }^{\gamma} G_{\gamma}, \\
\left\{G_{\alpha}, H_{0}\right\}_{D}=h_{\alpha}{ }^{\beta} G_{\beta},
\end{gathered}
$$

where equality is meant on the $\Theta=0$ surface. $f$ and $h$ are functions of the canonical variables. The action is given by

$$
S=\int d t\left(p_{i} \dot{q}^{i}-H_{0}-\lambda^{\alpha} G_{\alpha}\right)_{\Theta=0}
$$


where $\lambda^{\alpha}(t)$ are the Lagrange multipliers. $S$ is invariant under the gauge transformations

$$
\begin{aligned}
\delta p_{i} & =\epsilon^{\alpha}\left\{G_{\alpha}, p_{i}\right\}_{D} \\
\delta q^{i} & =\epsilon^{\alpha}\left\{G_{\alpha}, q^{i}\right\}_{D} \\
\delta \lambda^{\alpha} & =\dot{\epsilon}^{\alpha}-\lambda^{\beta} \epsilon^{\gamma} f_{\gamma \beta}{ }^{\alpha}-\epsilon^{\beta} h_{\beta}{ }^{\alpha} .
\end{aligned}
$$

It is clear from (6) that the $\lambda^{\alpha}$ play the role of the gauge fields corresponding to the symmetries generated by the $G_{\alpha}$.

Formula (5) together with (6) is exactly what one needs in order to construct a gauge invariant action based on a given algebra. The only non-trivial steps are finding the realization of the algebra in terms of the canonical variables, and obtaining $H_{0}$. This method was previously used by Siegel [7], to construct gauge invariant actions for the superparticle and the superstring. In the case of the $W$-algebras, obtaining the canonical representation turns out to be easy, although there is a subtlety in the choice of $H_{0}$ in the chiral case, related to the choice of the evolution parameter.

Since one would like to a have a scalar field theory action invariant under the $W$ gauge transformations, the canonical coordinates are then the two dimensional scalar fields $\phi_{i}(\sigma, \tau)$, where $i=1, \ldots, n$ and $\tau$ is the time $\left(\tau=x^{0}, \sigma=x^{1}\right)$. Let $P_{i}(\sigma, \tau)$ be the canonically conjugate momenta, satisfying

$$
\left\{P_{i}(\sigma, \tau), \phi_{j}\left(\sigma^{\prime}, \tau\right)\right\}=\delta_{i j} \delta\left(\sigma-\sigma^{\prime}\right)
$$

In order to construct the action, we need a canonical representation of a given Walgebra. This can be obtained from the free field representation of a $W$-algebra currents [4], by replacing $\partial_{ \pm} \phi_{i}$ with

$$
\hat{P}_{i}^{ \pm}=\frac{1}{\sqrt{2}}\left(P_{i} \pm \phi_{i}^{\prime}\right)
$$

where $f^{\prime}=\frac{\partial}{\partial \sigma} f$, and $f^{(n)}=\left(\frac{\partial}{\partial \sigma}\right)^{n} f$. Then the Poisson bracket alegbra of the $\hat{P}^{\prime} \mathrm{s}$ is isomorfic to that of the $\partial \phi$ 's

$$
\left\{\hat{P}_{i}^{ \pm}(\sigma, \tau), \hat{P}_{j}^{ \pm}\left(\sigma^{\prime}, \tau\right)\right\}=\mp \delta_{i j} \delta^{\prime}\left(\sigma-\sigma^{\prime}\right)
$$

Hence the generators of the $W_{N}$ algebra are given as

$$
W_{ \pm s}=\frac{1}{s} d_{i_{1} \ldots i_{s}} \hat{P}_{i_{1}}{ }^{ \pm} \ldots \hat{P}_{i_{s}}{ }^{ \pm} \quad(s=2, \ldots, N)
$$

The Poisson bracket algebra of the constraints (10) closes if the constants $d_{i_{1} \ldots i_{s}}$ satisfy certain algebraical relations [4],[8]. In the $W_{3}$ case these are

$$
d_{(i j \mid k} d^{k}{ }_{\mid l) m}=\kappa d_{(i j} d_{l) m}
$$


and the Poisson bracket algebra is

$$
\begin{aligned}
\left\{T_{ \pm}(\sigma), T_{ \pm}\left(\sigma^{\prime}\right)\right\} & =\mp \delta^{\prime}\left(\sigma-\sigma^{\prime}\right)\left(T_{ \pm}(\sigma)+T_{ \pm}\left(\sigma^{\prime}\right)\right) \\
\left\{T_{ \pm}(\sigma), W_{ \pm}\left(\sigma^{\prime}\right)\right\} & =\mp \delta^{\prime}\left(\sigma-\sigma^{\prime}\right)\left(W_{ \pm}(\sigma)+2 W_{ \pm}\left(\sigma^{\prime}\right)\right) \\
\left\{W_{ \pm}(\sigma), W_{ \pm}\left(\sigma^{\prime}\right)\right\} & =\mp 2 \kappa \delta^{\prime}\left(\sigma-\sigma^{\prime}\right)\left(T^{2}{ }_{ \pm}(\sigma)+T^{2}{ }_{ \pm}\left(\sigma^{\prime}\right)\right)
\end{aligned}
$$

where $T=W_{2}$ is the energy-momentum tensor and $W=W_{3}$.

In the non-chiral case the theory is diffeomorphism invariant, and therefore $H_{0}=$ 0 (otherwise, the wave functional $\Psi[\phi]$ would depend explicitely on the unphysical parameter $\tau$, since $\left.i \frac{\partial}{\partial \tau} \Psi=\hat{H}_{0} \Psi\right)$. Then according to (5) the gauge invariant action is simply

$$
S_{N}=\int d \sigma d \tau\left(P_{i} \dot{\phi}_{i}-h^{ \pm} T_{ \pm}-\sum_{s=3}^{N} B^{ \pm}{ }_{s} W_{ \pm s}\right)
$$

where $h$ and $B$ are the lagrange multipliers, which are also the gauge fields corresponding to the $W$-symmetries. The gauge transformation laws can be determined from (6). In the $W_{3}$ case they become

$$
\begin{aligned}
\delta P_{i} & =\frac{1}{\sqrt{2}}\left(\epsilon^{+} \hat{P}_{+i}-\epsilon^{-} \hat{P}_{-i}\right)^{\prime}+\frac{1}{\sqrt{2}}\left(\xi^{+} d_{i j k} \hat{P}_{+}^{j} \hat{P}_{+}^{k}-\xi^{-} d_{i j k} \hat{P}_{-}^{j} \hat{P}_{-}{ }^{k}\right)^{\prime}, \\
\delta \phi_{i} & =\frac{1}{\sqrt{2}} \epsilon^{ \pm} \hat{P}_{ \pm i}+\frac{\xi^{ \pm}}{\sqrt{2}} d_{i j k} \hat{P}_{ \pm}^{j} \hat{P}_{ \pm} k \\
\delta h^{ \pm} & =\dot{\epsilon}^{ \pm} \mp h^{ \pm}\left(\epsilon^{ \pm}\right)^{\prime} \pm\left(h^{ \pm}\right)^{\prime} \epsilon^{ \pm} \pm 2 \kappa\left(\xi^{ \pm}\left(B^{ \pm}\right)^{\prime}-\left(\xi^{ \pm}\right)^{\prime} B^{ \pm}\right) T_{ \pm} \\
\delta B^{ \pm} & =\dot{\xi}^{ \pm} \pm 2\left(h^{ \pm}\right)^{\prime} \xi^{ \pm} \mp h^{ \pm}\left(\xi^{ \pm}\right)^{\prime} \mp 2 B^{ \pm}\left(\epsilon^{ \pm}\right)^{\prime} \pm\left(B^{ \pm}\right)^{\prime} \epsilon^{ \pm}
\end{aligned}
$$

where we have taken $d_{i j}=\delta_{i j}$, and $\epsilon^{ \pm}$are the parameters of the $T_{ \pm}$transformations, while $\xi^{ \pm}$are the parameters of the $W_{ \pm}$transformations.

In order to find a geometrical interpretation of the action (13) we need to know it's second order form. It can be obtained by substituting in the expressions for the momenta $P_{i}$ obtained from the equation of motion

$$
\frac{\delta S}{\delta P_{i}}=0
$$

In the $W_{3}$ case one gets

$$
\dot{\phi}_{i}-\frac{1}{2} h^{ \pm}\left(P_{i} \pm \phi_{i}^{\prime}\right)-\frac{B^{ \pm}}{2 \sqrt{2}} d_{i j k}\left(P_{j} \pm \phi_{j}^{\prime}\right)\left(P_{k} \pm \phi_{k}^{\prime}\right)=0
$$

This is a quadratic equation in the $P$ 's, and therefore the second order form of the Lagrangian density will be a non-polynomial function of $\partial \phi, h$ and $B$, which can be written as an infinite power series in those variables. This will be a generic feature of 
all W-gravity actions, except for the $W_{2}$ case (usual 2 d gravity), where the equation (15) is linear in $P$ 's $(B$ indipendent part of $(16))$. This is analogous to the results of the Noether procedure, where a pair of auxilliary fields is introduced in order to have a closed form of the action, and they satisfy quadratic equations. However, the advantage of the Hamiltonian formalism is that one is automatically provided with the closed form of the action from the begining, and the momenta are the auxilliary fields. The standard drawback is the loss of manifest covariance.

As we have explained, the second order form of the action can be explicitely evaluated in the $W_{2}$ case

$$
S_{2}=\frac{1}{2} \int d^{2} \sigma \sqrt{-g} g^{\mu \nu} \partial_{\mu} \phi^{i} \partial_{\nu} \phi_{i}
$$

where

$$
\tilde{g}^{00}=\frac{2}{h^{+}+h^{-}} \quad, \quad \tilde{g}^{01}=\frac{h^{-}-h^{+}}{h^{+}+h^{-}} \quad, \quad \tilde{g}^{11}=-\frac{2 h^{+} h^{-}}{h^{+}+h^{-}},
$$

and $\tilde{g}^{\mu \nu}=\sqrt{-g} g^{\mu \nu}$. The geometrical interpretation of (17) is that it represents the action for $2 \mathrm{~d}$ gravity coupled to scalars. This can be verified by noticing first that the transformation law for a field $\phi_{i}((14 . b)$ with $\xi=0)$ can be written as

$$
\delta \phi_{i}=\epsilon^{ \pm} \tilde{e}_{ \pm}^{\mu} \partial_{\mu} \phi_{i}=\epsilon^{\mu} \partial_{\mu} \phi_{i}
$$

where

$$
\tilde{e}_{ \pm}^{\mu}=\frac{1}{h^{+}+h^{-}}\left(\begin{array}{cc}
1 & h^{-} \\
1 & -h^{+}
\end{array}\right) \text {. }
$$

Equation (19) is an infinitesimal diffeomorphism transformation of a scalar, where $\epsilon^{\mu}$ is the parameter of the transformation. Then one can show that the components of $\tilde{g}^{\mu \nu}$ given by (18) transform under the $\xi=0$ part of (14.c) as

$$
\delta \tilde{g}^{\mu \nu}=\partial_{\rho}\left(\epsilon^{\rho} \tilde{g}^{\mu \nu}\right)-\partial_{\rho} \epsilon^{(\mu \mid} \tilde{g}^{\mid \nu) \rho},
$$

which is the diffeomorphism transformation of a densitized metric generated by the parameter $\epsilon^{\mu}$. The metric $g^{\mu \nu}$ can be written as

$$
g^{\mu \nu}=\frac{\rho}{\left(h^{+}+h^{-}\right)^{2}}\left(\begin{array}{cc}
2 & h^{-}-h^{+} \\
h^{-}-h^{+} & -2 h^{+} h^{-}
\end{array}\right)=e_{+}{ }^{(\mu \mid} e_{-}{ }^{\mid \nu)},
$$

where $e_{ \pm}^{\mu}=\sqrt{\rho} \tilde{e}_{ \pm}{ }^{\mu}$ are the zweibeins and $\rho$ is the conformal mode of the metric. Note that the action (17) is indipendent of $\rho$ due to the Weyl symmetry

$$
\delta g^{\mu \nu}=\omega g^{\mu \nu}
$$

so that

$$
\sqrt{-g}=|e|=\frac{h^{+}+h^{-}}{\rho}
$$


In the $W_{3}$ case the geometric interpretation is not obvious due to the lack of the explicit form of the second order action. In order to obtain the second order form of the action we will rewrite the momentum equation (16) as

$$
\begin{aligned}
P_{i} & =\frac{2}{h^{+}+h^{-}}\left[\dot{\phi}_{i}-\frac{1}{2}\left(h^{+}-h^{-}\right) \phi_{i}^{\prime}-\frac{B^{ \pm}}{\sqrt{2}} d_{i j k} \hat{P}_{ \pm}^{j} \hat{P}_{ \pm}{ }^{k}\right] \\
& =P_{i}{ }^{0}+P_{i}{ }^{1}
\end{aligned}
$$

where $P_{i}^{0}$ is the $B$ indipendent part of (25). Then the action (13) takes the following form

$$
S_{3}=\int d^{2} \sigma\left(|e| \partial_{+} \phi_{i} \partial_{-} \phi_{i}-\frac{h^{+}+h^{-}}{4}\left(P_{i}^{1}\right)^{2}-\frac{B^{ \pm}}{3} d_{i j k} \hat{P}_{ \pm}^{i} \hat{P}_{ \pm}^{j} \hat{P}_{ \pm}^{k}\right)
$$

where $\partial_{ \pm}=e_{ \pm}{ }^{\mu} \partial_{\mu} . \hat{P}$ satisfies

$$
\hat{P}_{ \pm i}=\sqrt{2} \tilde{\partial}_{ \pm} \phi_{i}-\frac{B^{ \pm}}{h^{+}+h^{-}} d_{i j k} \hat{P}_{ \pm}^{j} \hat{P}_{ \pm}^{k}
$$

where $\tilde{\partial}=\rho^{-\frac{1}{2}} \partial$. By using (27) one can obtain the power series expansion of $\hat{P}$ in terms of $\partial_{ \pm} \phi$ and $B$, which can be inserted into (26) to give the corresponding power series expansion of the action. Up to the first order in $B$ the Lagrange desity can be written as

$$
\mathcal{L}=|e|\left(\partial_{+} \phi_{i} \partial_{-} \phi_{i}-B^{ \pm \pm \pm} d_{i j k} \partial_{ \pm} \phi^{i} \partial_{ \pm} \phi^{j} \partial_{ \pm} \phi^{k}\right)+O\left(B^{2}\right)
$$

where

$$
B^{ \pm \pm \pm}=\frac{2 \sqrt{2}}{3} \frac{B^{ \pm}}{\sqrt{\rho}\left(h^{+}+h^{-}\right)}
$$

Equation (28) suggests the geometrical interpretation based on the zweibeins introduced in the $W_{2}$ case. However, that is not possible, since the second order in $B$ contribution to $\mathcal{L}$ is

$$
\mathcal{L}_{(2)}=\frac{9}{4}|e|\left(B^{+++} d_{i j k} \partial_{+} \phi^{j} \partial_{+} \phi^{k}+B^{---} d_{i j k} \partial_{-} \phi^{j} \partial_{-} \phi^{k}\right)^{2}
$$

which is not diffeomorphism invariant. The reason for this is that the \pm indicies in (28) and (30) are not covariant because the diffeomorphism transformation of $\phi_{i}$ in the $W_{3}$ case is not $\delta \phi_{i}=\epsilon^{ \pm} \tilde{\partial}_{ \pm} \phi_{i}$ but

$$
\delta \phi_{i}=\frac{1}{\sqrt{2}} \epsilon^{ \pm} \hat{P}_{ \pm i}=\epsilon^{ \pm}\left(\tilde{\partial}_{ \pm} \phi_{i}-\frac{\sqrt{2} B^{ \pm}}{\left(h^{+}+h^{-}\right)} d_{i j k} \tilde{\partial}_{ \pm} \phi^{j} \tilde{\partial}_{ \pm} \phi^{k}+\cdots\right)
$$

Note that the equation (31) can be rewritten as

$$
\delta \phi_{i}=\epsilon^{\mu} \partial_{\mu} \phi_{i} \quad, \quad \epsilon^{\mu}=f^{\mu}\left(\epsilon^{ \pm}, h^{ \pm}, B^{ \pm}, \partial_{ \pm} \phi\right)
$$


The function $f$ is not unique, and the difficulty at the moment is to see which choice of $f$ is the right one. However, given that the form (32) exists, and that $\mathcal{L}$ can be written as

$$
\begin{aligned}
\mathcal{L}= & \frac{1}{2} \tilde{g}^{\mu \nu} \partial_{\mu} \phi^{i} \partial_{\nu} \phi_{i}+\tilde{B}^{\mu \nu \rho} d_{i j k} \partial_{\mu} \phi^{i} \partial_{\nu} \phi^{j} \partial_{\rho} \phi^{k} \\
& +\tilde{C}^{\mu \nu \rho \eta} d_{i j}{ }^{m} d_{m k l} \partial_{\mu} \phi^{i} \partial_{\nu} \phi^{j} \partial_{\rho} \phi^{k} \partial_{\eta} \phi^{l}+\cdots,
\end{aligned}
$$

one can argue that the objects $\tilde{g}, \tilde{B}, \tilde{C}, \ldots$, must transform as tensor densities in order for (33) to be invariant under (32). As far as the zweibein interpretation is concerned, the formula (32) gives a clue. Namely, one can write

$$
\epsilon^{\mu}=f^{\mu}\left(\epsilon^{ \pm}, h^{ \pm}, B^{ \pm}, \partial_{ \pm} \phi\right)=\epsilon^{ \pm} \tilde{e}_{ \pm}^{\mu}\left(h^{ \pm}, B^{ \pm}, \partial_{ \pm} \phi\right)
$$

which means that the zweibeins (20) change in the $W_{3}$ case. They become functions of $B$ and $\partial \phi$ such that when $B \rightarrow 0$ then $\tilde{e}(h, B, \partial \phi) \rightarrow \tilde{e}(h)$ of $(20)$.

Besides the diffeomorphism invariance, the generalized Weyl symmetry [4],[9] is also obscured. Heuristically, it is there by construction, since we used only four indipendent gauge fields $h^{ \pm}$and $B^{ \pm}$. The fields $\tilde{g}, \tilde{B}, \tilde{C}, \ldots$ in (33) are functions of $h$ and $B$, and one can check order by order in $\partial \phi$ that

$$
\tilde{g}_{\mu \nu} \tilde{B}^{\mu \nu \rho}=0 \quad, \quad \tilde{C}^{\mu \nu \rho \sigma}=\tilde{g}_{\chi \eta} \tilde{B}^{\chi \mu \nu} \tilde{B}^{\eta \rho \sigma},
$$

and so on. The generalized Weil invariance of the complete action follows from the results obtained recently by Hull in the context of the $W_{\infty}$ gauge theory [9]. Namely, a Lagrange density $\mathcal{L}(x, \phi(x), \partial \phi(x))$ is invariant under the generalized Weyl symmetry if the function

$$
\tilde{F}(x, y)=\left.\mathcal{L}(x, \phi(x), \partial \phi(x))\right|_{\partial \phi=y}
$$

satisfies the Monge-Ampere equation

$$
\operatorname{det}\left|\frac{\partial^{2} \tilde{F}(x, y)}{\partial y_{\mu} \partial y_{\nu}}\right|=-1
$$

One can show that

$$
\tilde{F}(x, y)=P \dot{\phi}-f\left(x, P+\phi^{\prime}\right)-\bar{f}\left(x, P-\phi^{\prime}\right)
$$

is a general solution of (37), where $P$ is the auxilliary field determined by $P=$ $\frac{\partial \tilde{F}}{\partial \dot{\phi}}$, and $f$ and $\bar{f}$ are arbitrary functions. Note that the expression (38) is exactly the hamiltonian form of the Lagrange density for a $W$-gravity theory (13), and the functions $f$ and $\bar{f}$ are just the linear combinations of the constraints (10). 
It is clear that given any extended conformal algebra realized in terms of free scalar fields, the gauge invariant action can be written down imidiately by following the procedure we have presented. Even when the classical Poisson bracket algebra of the constraints contains a central extension, the method still works, since then the central charge can be treted as an extra Abelian generator, and the formula (5) would still apply. In that case the free field expressions for the currents will contain the higher derivative terms $\partial^{n} \phi$ [8], which would be replaced by $\hat{P}^{(n-1)}$ in order to obtain the constraints. Note that this construction is very similar to the corresponding Noether construction in terms of the auxilliary $F$-fields [6], where $\partial^{n} \phi$ is replaced with $\partial^{n-1} F$. However, there is a substantial difference. While the Hamiltonian action will still be first order in time derivatives, the Noether action will contain higher order time derivatives. This might be an indication that in the case when the central charges (or equivalently the background charges) are present, the two methods give different actions. This requires a further investigation.

Inclussion of the fermions in the Hamiltonian formalism is straightforward. They are represented as the Grassman valued fields $\psi^{ \pm}{ }_{a}(\sigma, \tau)$, which are canonically selfconjugate

$$
\left\{\psi^{ \pm}{ }_{a}(\sigma, \tau), \psi_{b}^{ \pm}\left(\sigma^{\prime}, \tau\right)\right\}_{D}=\delta_{a b} \delta\left(\sigma-\sigma^{\prime}\right)
$$

The Hamiltonian constraints associated with a given extended conformal algebra can be obtained from a free-field representation by replacing $\partial_{ \pm}^{n} \phi$ with $\hat{P}^{(n-1)} \pm$ and $\partial_{ \pm}{ }^{n} \psi_{ \pm}$with $\psi^{(n)}{ }_{ \pm}$, so that the corresponding gauge invariant action is

$$
S_{G}=\int d \sigma d \tau\left[P_{i} \dot{\phi}_{i}+\frac{1}{2} \psi^{ \pm}{ }_{a} \dot{\psi}^{ \pm}{ }_{a}-\mathcal{B}^{ \pm}{ }_{\alpha} G_{ \pm \alpha}\left(\hat{P}_{ \pm}, \hat{P}_{ \pm}^{\prime}, \ldots, \psi_{ \pm}, \psi_{ \pm}^{\prime}, \ldots\right)\right]
$$

where $G_{ \pm \alpha}$ are the left/right currents, satisfying (3), and $\mathcal{B}^{ \pm}{ }_{\alpha}$ are the corresponding gauge fields (Lagrange multipliers). The gauge transformations can be determined from (6).

Our final comment concernes the chiral case. As shown in [6], the Noether form of the chiral action [1],[4], follows directly from the formula (5) if the evolution parameter is chosen to be $x^{-}$. In the case when $x^{0}$ is chosen as the evolution parameter, the form of the chiral action changes. By analysing the $W_{2}$ case one can see that a non-zero $H_{0}$ appears

$$
H_{0}=-\int d \sigma P_{i} \phi_{i}^{\prime}
$$

which is a consequence of the fact that the chiral theory is not diffeomorphism invariant (i.e. only a "half" of the diffeomorphism invariance is present). Since

$$
\left\{W_{+s}(\sigma), H_{0}\right\}=\frac{\partial}{\partial \sigma} W_{+s}(\sigma)
$$


so that (4) is satisfied, the action is given by (5)

$$
S_{N}^{(c h)}=\int d \sigma d \tau\left(P_{i} \dot{\phi}_{i}+P_{i} \phi_{i}^{\prime}-h^{+} T_{+}-\sum_{s=3}^{N} B_{s}^{+} W_{+s}\right) .
$$

The gauge transformations can be read off from (6). For $N \geq 3$ the second order form of this action appears to be nonpolynomial, in contrast to the polynomial chiral Noether action. However, one can expect from the general arguments that the Hamiltonian form of the chiral Noether action is precisely (43), which implies that the second order form of (43) is polynomial.

I would like to thank Chris Hull for helpfull discussions.

\section{REFERENCES}

[1] C.M. Hull, Phys. Lett. 240B (1990) 110

[2] K. Schoutens, A. Sevrin and P. van Nieuwenhuizen, Phys. Lett. 243B (1990) 245

[3] E. Bergshoeff, C.N. Pope, L.J. Romans, E. Sezgin, X. Shen and K.S. Stelle, Phys. Lett. 243B (1990) 350

[4] C.M. Hull, Nucl. Phys. B353 (1991) 707

[5] C.M. Hull, Phys. Lett. 259B (1991) 68

[6] A. Miković, Phys. Lett. 260B (1991) 75

[7] W. Siegel, Nucl. Phys. B263 (1985) 93

[8] L.J. Romans, Nucl. Phys. B352 (1991) 829

[9] C.M. Hull, The geometry of W-gravity, QMWC preprint (1991) QMW/PH/91/6 